\title{
Die Kommunikationsstrategien der Kontrahenten in der Fluglärmdebatte. Einflüsse der Wahrnehmung einer feindlichen Berichterstattung, der Medienwirksamkeit und der Meinungsklimata in Politik und Öffentlichkeit
}

\author{
Post, Senja
}

DOI: https://doi.org/10.5771/1615-634X-2015-4-517

Posted at the Zurich Open Repository and Archive, University of Zurich ZORA URL: https://doi.org/10.5167/uzh-117934

Journal Article

Published Version

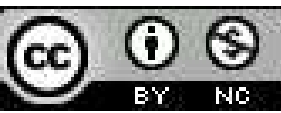

The following work is licensed under a Creative Commons: Attribution-NonCommercial 4.0 International (CC BY-NC 4.0) License.

Originally published at:

Post, Senja (2015). Die Kommunikationsstrategien der Kontrahenten in der Fluglärmdebatte. Einflüsse der Wahrnehmung einer feindlichen Berichterstattung, der Medienwirksamkeit und der Meinungsklimata in Politik und Öffentlichkeit. Medien Kommunikationswissenschaft:517-534.

DOI: https://doi.org/10.5771/1615-634X-2015-4-517 


\title{
Die Kommunikationsstrategien der Kontrahenten in der Fluglärmdebatte
}

\begin{abstract}
Einflüsse der Wahrnehmung einer feindlichen Berichterstattung, der Medienwirksamkeit und der Meinungsklimata in Politik und Öffentlichkeit*
\end{abstract}

Senja Post

Nach dem Modell der reziproken Effekte wirkt die Berichterstattung über Konflikte auf die Konfliktgegner zurück und beeinflusst ibr Kommunikationsverbalten und damit die Konfliktdynamik. Als hochinvolvierte Akteure nebmen die Konfliktgegner die Berichterstattung i.d.R. als feindlich verzerrt gegenüber dem eigenen Standpunkt wahr (Hostile Media Phenomenon). Nach der "Corrective-Actions"-Hypothese (Rojas 2010) mobilisiert diese Wabrnebmung zu Versuchen, die vermeintlich feindliche Berichterstattung durch kommunikatives Engagement auszugleichen. Diese Annabme wird am Beispiel der Konfliktgegner in der deutschen Fluglärmdebatte basierend auf einer Online-Befragung der Fluglärmgegner $(n=82 ; 47 \%)$ und Befürworter des Luftverkebrs $(n=48$; $33 \%$ geprüft. Für die Befürworter des Luftverkehrs bestätigt sich die Hypothese über die korrigierenden Handlungen: Sie engagieren sich vor allem dann kommunikativ, wenn sie sich mit einer feindlich gesinnten Berichterstattung und einem geringen Medieninteresse konfrontiert sehen. Für die Fluglärmgegner bestätigt sich die Hypothese nicht: Sie engagieren sich vor allem dann, wenn sie ein hohes Medieninteresse wabrnebmen.

Schlüsselwörter: Hostile Media Phänomen, Corrective Actions, reziproke Effekte, vermutete Medienwirkungen, publizistische Konflikte, Online-Befragung

\section{Einleitung}

Medienberichte wirken nicht nur auf die Masse ihrer Leser, Hörer oder Zuschauer, sondern auch auf diejenigen, über die die Medien berichten. Diese sogenannten reziproken Effekte (Kepplinger 2007) können ihrerseits weitreichende Konsequenzen haben. Beispiele sind Politiker, die sich aufgrund der Berichterstattung bestimmten politischen Sachthemen zuwenden; Richter, die aufgrund der Berichterstattung über ihren Prozess bestimmte Argumente in ihrem Urteil berücksichtigen (Kepplinger \& Zerback 2012); Wissenschaftler, die sich aufgrund des Medieninteresses bestimmten Forschungsfragen zuwenden (Post 2009) usw. Bislang wurden reziproke Effekte der Medien allgemein auf die Protagonisten der Berichterstattung untersucht - auf diejenigen Personen oder Angehörigen von Gruppen, über die die Medien berichten. Dagegen gibt es bislang kaum Studien über die reziproken Effekte in Konflikten - die Wirkungen der Konfliktberichterstattung auf die gegnerischen Parteien bzw. die Antagonisten.

Nach dem Modell der reziproken Effekte nehmen die Protagonisten, bzw. hier die Antagonisten, die Berichterstattung über sich selbst oder die Gruppen, denen sie angehören, wahr, stellen Überlegungen über die Wirkungen der Berichterstattung an und fällen auf dieser Grundlage ihre Handlungsentscheidungen - bspw. über ihre öffentliche Kommunikation (Kepplinger 2007). Die Gegner in Konflikten nehmen die Berichter-

* Die Autorin dankt drei anonymen Gutachtern, von denen sich vor allem einer außergewöhnlich intensiv mit dem Manuskript beschäftigt hat, für viele wertvolle Hinweise auf Verbesserungsmöglichkeiten des Manuskripts. 
stattung in der Regel als feindlich verzerrt gegenüber ihrem Standpunkt wahr (Hostile Media Perception, Vallone, Ross \& Lepper 1985). Einige Studien zeigen, dass die Wahrnehmung einer feindlichen Berichterstattung mobilisieren kann - bspw. zu einem erhöhten Engagement in sozialen Netzwerken, auf Demonstrationen, in Initiativen usw. (Barnidge \& Rojas 2014; Feldman et al. 2015; Hwang, Pan \& Sun 2008; Rojas 2010). Dieser Zusammenhang wurde bei den Anbängern von Konfliktlagern belegt - also bspw. in den weiten Kreisen der Befürworter und Kritiker von Konfliktgegenständen, oder bei den Wählern gegnerischer politischer Parteien. Dagegen gibt es bislang kaum Untersuchungen, die die Auswirkungen der Wahrnehmung einer feindlichen Berichterstattung auf das Kommunikationsverhalten der Konfliktgegner im engeren Sinne analysieren auf das Verhalten derjenigen, die als Wortführer, Koordinatoren, Organisatoren oder Sprecher die Interessen ihrer Partei bündeln, ihre Mitglieder mobilisieren, nach außen vertreten und die Verhandlungs- und Kommunikationsstrategien festlegen. Die Medienwirkungen der Konfliktberichterstattung auf den engen Kreis der führenden Konfliktgegner sind gesellschaftlich besonders relevant, weil sie erhebliche Auswirkungen auf die öffentliche Auseinandersetzung, die Konfliktdynamik, den Zulauf oder die Abkehr von Unterstützern oder die Kompromissbildung haben dürften.

In der vorliegenden Untersuchung geht es um reziproke Effekte der Berichterstattung über die Debatte um den Fluglärm auf die beteiligten Konfliktgegner im oben genannten Sinn: die Wortführer, Koordinatoren, Organisatoren oder Sprecher auf Seiten der Fluglärmgegner und auf Seiten der Befürworter des Luftverkehrs. Konkret soll geprüft werden, wie die Konfliktgegner die Berichterstattung über den Fluglärmstreit wahrnehmen und inwieweit ihre Wahrnehmungen der Berichterstattung und Vermutungen von Medienwirkungen ihre Kommunikationsstrategien beeinflussen.

\section{Theoretische Grundlagen: Die Wahrnehmung feindlicher Medieninhalte und ihre Einflüsse auf das kommunikative Engagement der Anhänger von Konfliktlagern}

Vallone, Ross und Lepper (1985) entdeckten das sogenannte Hostile Media Phenomenon, indem sie US-amerikanischen Studenten einen ausgewogenen Artikel über das Beirut-Massaker von 1982 vorlegten und sie nach ihrer Bewertung des Artikels fragten. Versuchspersonen mit pro-israelischer Haltung hielten den Artikel für pro-palästinensisch, Versuchspersonen mit pro-palästinensischer Haltung für pro-israelisch. Das Hostile Media Phenomenon wurde durch zahlreiche Experimente und Befragungsstudien in unterschiedlichen Kontexten belegt - in generellen politisch-ideologischen Konflikten (Barnidge \& Rojas 2014; Coe et al. 2008; Rojas 2010) und in spezifischeren Kontexten wie z. B. im Wahlkampf (Huge \& Glynn 2010), in der Kontroverse um die jüdischen Siedlungen im Gaza-Streifen (Tsfati \& Cohen 2005) sowie in den US-amerikanischen Debatten über Abtreibung (Hartmann \& Tanis 2013), Stammzellforschung (Ho et al. 2011) oder den Klimawandel (Feldman et al. 2015).

Als Ursachen für die Wahrnehmung einer feindlichen Berichterstattung gelten auf Seiten der Rezipienten der Grad ihrer Involvierung in das Konfliktthema (Gunther, Miller \& Liebhart 2009; Choi, Yang \& Chang 2009; Matthes 2013) und die Extremität ihrer Einstellung (Hwang et al. 2008). Auch die Identifikation mit einer Konfliktpartei bzw. die Gruppenzugehörigkeit (Hartmann \& Tanis 2013; Reid 2012; Stroud, Muddiman \& Lee 2014) und der wahrgenommene Status der eigenen Konfliktpartei (Hartmann \& Tanis 2013) sind wichtige Erklärungsfaktoren. Auf Seiten der Botschaft spielt beispielsweise die Glaubwürdigkeit der Quelle und die vermutete Reichweite der Botschaft eine wichtige Rolle: Menschen nehmen Botschaften vor allem dann als feindlich wahr, 
wenn sie mit einer hohen, weniger dagegen, wenn sie mit einer geringen Reichweite rechnen (Gunther \& Schmitt 2004; Gunther \& Liebhart 2006).

In den vergangenen Jahren gerieten vermehrt die Konsequenzen des Hostile Media Phenomenon in den Blick der Forschung. Mehrere Studien belegen, dass die Wahrnehmung einer feindlichen Berichterstattung das Kommunikations- oder Partizipationsverhalten der Anhänger von Konfliktlagern intensivieren kann - z. B. ihre Teilnahme an Demonstrationen, Unterschriftenaktionen, ihre Verbreitung von Nachrichten in sozialen Netzwerken usw. (Hwang et al. 2008; Feldman et al. 2015). Diese Aktivitäten können als „korrigierende Handlungen“ (Rojas 2010) gedeutet werden - als Versuche, der vermeintlich negativen Berichterstattung durch Kommunikationsaktivitäten oder politisches Engagement entgegenzuwirken (Barnidge \& Rojas 2014; Bernhard \& Dohle 2013; Feldman et al. 2015).

Nach dem Modell der Persuasive Press Inference (Gunther 1998) gehen Menschen davon aus, dass die wahrgenommene Berichterstattung wirkungsvoll ist und sich auf die öffentliche Meinung niederschlägt. Tatsächlich bestätigen mehrere Studien, dass für das Kommunikations- und Partizipationsverhalten der Anhänger von Konfliktlagern neben der Wahrnehmung einer feindlichen Berichterstattung auch Vermutungen von Medieneinflüssen (Barnidge \& Rojas 2014; Bernhard \& Dohle 2013; Rojas 2010; Tsfati \& Cohen 2005) und die Wahrnehmung relevanter Meinungsklimata ausschlaggebend sein können (Tsfati 2007; Tsfati \& Cohen 2005). Hierzu ist die Befundlage allerdings nicht eindeutig: Einige Studien zeigen, dass die Anhänger von Konfliktlagern umso intensiver bzw. offensiver kommunizieren und sich engagieren, je stärkere Wirkungen der Berichterstattung sie erwarten (Barnidge \& Rojas 2014; Rojas 2010) und je mehr sie die öffentliche Meinung gegen sich sehen (Tsfati \& Cohen 2005). Solche Effekte zeigten sich dagegen nicht bei der wahlberechtigten Bevölkerung Baden-Württembergs im Kontext des Konflikts um Stuttgart 21 (Bernhard \& Dohle 2013). Andere Studien zeigen sogar gegenteilige Einflüsse: So fanden Bernhard und Dohle (2015) im Kontext des Bundestagswahlkampfes 2013, dass die Wahrnehmung einer negativen und einflussreichen TV-Berichterstattung die Bereitschaft der Wähler gesenkt hat, ihre Meinung im Internet zu verbreiten. Die Wähler verbreiteten ihre Meinung im Internet vor allem dann, wenn sie sich durch die TV-Nachrichten bestätigt fühlten. Am Beispiel der arabischen Minderheit in Israel zeigte Tsfati (2007), dass die Wahrnehmung einer feindlichen Berichterstattung und eines feindlichen Meinungsklimas nicht zu einer Mobilisierung, sondern zur sozialen Entfremdung führt.

Mögliche Erklärungen für diese Unterschiede dürften spezifische Merkmale der untersuchten Gruppen sein, bspw. die Homogenität der Konfliktparteien, die Extremität ihrer Forderungen oder die Wahrnehmung ihres gesellschaftlichen Status durch die Angehörigen (Hartmann \& Tanis 2013). Relevant ist außerdem die Selbstwirksamkeit der Angehörigen von Konfliktlagern: Feldman et al. (2015) zeigten im Kontext der US-Debatte über den Klimawandel, dass die Bereitschaft zu politischem Engagement als Reaktion auf eine vermeintlich feindliche Berichterstattung in der Bevölkerung umso größer ist, je mehr die Menschen den Eindruck haben, dass sie den politischen Prozess beeinflussen können.

Erstaunlicherweise spielte in keiner der erwähnten Studien zum Einfluss des Hostile Media Phenomenon auf das politische oder kommunikative Engagement die Medienwirksamkeit eine Rolle - der Eindruck, mit den eigenen Positionen in den Medien durchdringen zu können. Zwar wurden in den erwähnten Studien verschiedene Aspekte der Wahrnehmung einer feindlichen Berichterstattung erfasst - die wahrgenommene Position der Medien für oder gegen einen Konfliktgegenstand (Post 2015); die Distanz 
der eigenen zur Position der Medien (Barnidge \& Rojas 2014; Rojas 2010); der Eindruck, dass die Medien das eigene Konfliktlager negativ darstellen (Tsfati 2007), feindlich verzerrt gegenüber der eigenen Position berichten, oder von gegnerischen Interessengruppen beeinflusst sind (Feldman et al. 2015; Hwang et al. 2008). Unberücksichtigt blieb jedoch bislang die Frage der wahrgenommenen Einflussmöglichkeit - die Selbstwirksamkeit gegenüber den Medien. Man kann vermuten, dass eine geringe Medienwirksamkeit das Partizipationsverhalten der Anhänger von Konfliktlagern in ähnlicher Weise beeinflusst wie die Wahrnehmung, dass die Medien feindlich über die eigene Position berichten. In der vorliegenden Untersuchung soll das geprüft werden.

\section{Korrigierende Handlungen von Konfliktgegnern}

Die meisten Studien zum Einfluss des Hostile Media Phenomenon auf das Kommunikations- und Partizipationsverhalten basieren auf Repräsentativbefragungen der allgemeinen Bevölkerung (Feldman et al. 2015; Bernhard \& Dohle 2013; Bernhard \& Dohle 2015). Gegenstand dieser Untersuchungen sind die Angehörigen von Konfliktlagern diejenigen, die der einen oder anderen Partei in einem Konflikt mehr oder weniger zugeneigt sind und sie mehr oder weniger aktiv unterstützen. Gegenstand der vorliegenden Studie sind hingegen die Konfliktgegner im engeren Sinne - diejenigen Personen, die die Interessen einer Konfliktpartei offiziell in Verbänden, Vereinen, Bürgerinitiativen, Allianzen oder anderen Gruppierungen organisieren, sie nach außen vertreten und durchzusetzen versuchen, somit auch als öffentliche Sprecher, d. h. als potentielle Medienquellen fungieren. Die Konfliktgegner im engeren Sinne sind Personen, die die öffentliche Debatte wesentlich prägen und daher auch die Konfliktdynamik beeinflussen.

In vielen Konflikten - wie auch im hier untersuchten Streit um den Fluglärm - stehen sich ungleiche Konfliktgegner gegenüber. Kriesi (2001) unterscheidet idealtypisch etablierte politische Akteure (Insider) und Außenseiter. Beide wählen typischerweise unterschiedliche Kommunikationsstrategien, um ihre Interessen durchzusetzen. Unter die etablierten politischen Akteure fasst Kriesi Regierung, Opposition, Parteien, Interessenverbände, Verwaltungseinheiten. Sie haben aufgrund langfristig etablierter Kontakte in die Politik mehr oder weniger direkten Zugang zu politischen Entscheidungsprozessen. Unter die Außenseiter fasst er Organisationen sozialer Bewegungen (ebd.: 9). Sie haben keinen direkten Zugang zu politischen Entscheidungsprozessen, sondern können zunächst nur indirekt über die Medien und die Öffentlichkeit Einfluss auf Entscheidungsprozesse nehmen (Torgersen \& Hampel 2012). Die Herstellung von Öffentlichkeit ist für die Außenseiter daher eine wichtige Vorrausetzung dafür, Gehör in der Politik zu finden.

In den meisten Studien zum Einfluss des Hostile Media Phenomenon auf die Kommunikations- und Partizipationsbereitschaft der Anhänger von Konfliktlagern wurden vielzählige Aktivitäten erfasst, die man in Anlehnung an Kriesi (2001) als Außenseitertaktiken bezeichnen kann: Aktivitäten, die nicht auf einen direkten Kontakt zur Politik, sondern auf die Herstellung von Öffentlichkeit zielen. Hierzu zählen Aktivitäten zur Verbreitung von Informationen in der Bevölkerung sowie medienwirksame Protestaktionen („Informationspolitik“ und „Protestpolitik“, ebd.: 22ff.). In vorangegangenen Studien (Bernhard \& Dohle 2013, 2015; Feldman et al. 2015; Hwang et al. 2008; Rojas 2010) wurden als Informationsaktivitäten bspw. die Häufigkeiten der Nutzung von Flugblättern, Leserbriefen, E-Mails, sozialen Netzwerken, Blogs sowie Kommentarfunktionen auf Online-Nachrichtenseiten und als Demonstrationsaktivitäten bzw. Aktivismus bspw. die Teilnahme an Unterschriftenaktionen, Demonstrationen oder Freiwilligenarbeit in einer Initiative erhoben. Daneben wurden oft auch Aktivitäten erhoben, 
die nicht auf die Herstellung von Öffentlichkeit zielen, wie persönliche Gespräche (Barnidge \& Rojas 2014; Bernhard \& Dohle 2013; Rojas 2010). Dagegen wurden nur selten Aktivitäten abgefragt, die auf direkte Kontakte zu Politikern zielen. Feldman et al. (2015) fragten in ihrer Studie zwar nach der Häufigkeit von E-Mails oder Briefen an bzw. Anrufen bei Politikern. Allerdings bleibt bei diesen Informationen offen, ob es sich um Austauschbeziehungen oder einseitige Kontaktaufnahmen handelt. Einzig Hwang et al. (2008) fragten nach der Häufigkeit von Treffen mit Politikern - einer Aktivität, die vermutlich vorrangig den Angehörigen etablierter Interessen vorbehalten ist. Die erwähnten Studien differenzieren zudem nicht zwischen den beschriebenen Typen von Außenseiter- und Insider-Aktivitäten, sondern fassen sie in übergreifenden Indizes zusammen. ${ }^{1}$ Für eine Analyse des Kommunikations- und Partizipationsverhaltens der Anhänger von Konfliktlagern ist dies unproblematisch. Für eine Untersuchung der engeren Kreise der Gegner aus unterschiedlichen Konfliktparteien scheint es dagegen notwendig, einerseits die Insidertaktiken stärker zu berücksichtigen (Lobbying) und andererseits zwischen Außenseiter- (Verbreitung von Information in der Öffentlichkeit, Demonstrationsaktivitäten) und Insideraktivitäten zu unterscheiden.

\section{Die Gegner im Konflikt um den Fluglärm}

Ziel der vorliegenden Untersuchung ist es, den Einfluss der Wahrnehmung einer feindlichen Berichterstattung, vermuteter Medienwirkungen, relevanter Meinungsklimata sowie der Medienwirksamkeit auf das kommunikative und politische Engagement der Gegner im Konflikt um den Fluglärm an deutschen Flughäfen zu prüfen. Über die Lärmbelastung durch den Luftverkehr wird bundesweit an deutschen Flughäfen gestritten. Ein wichtiges Gebiet in diesem Konflikt ist die Region um den Frankfurter Flughafen. Daneben gibt es Protest gegen Fluglärm auch in den Gebieten um die internationalen Flughäfen München, Köln/Bonn, Düsseldorf, Hamburg, Stuttgart und Nürnberg sowie um einige Regionalflughäfen (z. B. Bremen und Dortmund). Die Fluglärmgegner argumentieren, dass der Fluglärm die Gesundheit der Anwohner und die Umwelt gefährdet. Sie fordern deshalb u. a. den Stopp des Ausbaus von Flughäfen sowie ein bundesweites Nachtflugverbot zwischen 22 und 6 Uhr. Die Befürworter des Luftverkehrs (Unternehmen der Luftverkehrswirtschaft sowie direkt davon abhängige Industrien wie Post- und Kurierdienste, Logistik- und Frachtunternehmen) argumentieren, dass eine gute Luftinfrastruktur ein wichtiger nationaler Wirtschaftsfaktor ist und viele Arbeitsplätze schafft. Sie fordern daher den bedarfsgerechten Ausbau der Flughäfen und die Ausweitung der Flugzeiten.

Die Befürworter des Luftverkehrs sind in einflussreichen Verbänden organisiert, wie dem Bundesverband der Deutschen Luftverkehrswirtschaft (BDL), der Arbeitsgemeinschaft deutscher Flughäfen (ADV) oder dem Bundesverband der Deutschen Fluggesellschaften (BDF). Sie unterhalten aufgrund einer Vielzahl von Themen (Luftverkehrssteuer, Emissionshandel u. v. a. m) vielfältige Kontakte zur Politik. Die Fluglärmgegner organisieren sich lokal in zahlreichen Bürgerinitiativen, die deutschlandweit sehr gut vernetzt sind. 1972 hat sich die Protestbewegung in der Bundesvereinigung gegen Fluglärm (BVF) zusammengeschlossen. An verschiedenen Flughäfen - z. B. in Frankfurt und München - demonstrieren die Fluglärmgegner regelmäßig. An anderen Flughäfen finden unregelmäßige Demonstrationen statt. Aufgrund ihres Organisationsgrades und ihrer langjährigen Aktivität dürften die Fluglärmgegner mittlerweile nicht mehr nur auf klas-

1 Rojas (2010) sowie Bernhard \& Dohle (2013, 2015) differenzieren - für ihr Forschungsinteresse angemessen - zwischen online- und offline-Aktivitäten. 
sische Außenseitertaktiken setzen, sondern auch zahlreiche direkte Kontakte in die Politik pflegen. Umgekehrt beschränken sich die Befürworter des Luftverkehrs nicht auf Insider-Politik, sondern versuchen, ihre Standpunkte auch durch Protest- und Informationspolitik in der Medienöffentlichkeit zu kommunizieren. Ein markantes Beispiel hierfür war die Demonstration „Ja zu FRA“ in der Frankfurter Innenstadt, zu der der Frankfurter Flughafenbetreiber Fraport sowie die Airlines Lufthansa und Condor am 1.3.2012 aufgerufen hatten. Welche Bedeutung die verschiedenen Taktiken für die generellen Kommunikationsstrategien der Konfliktgegner besitzen, ist allerdings unklar.

\section{Annahmen der Untersuchung}

Aus den Befunden zum Einfluss der Wahrnehmung einer feindlichen Berichterstattung auf das kommunikative und politische Engagement der Anhänger von Konfliktlagern kann man die folgenden Annahmen über das kommunikative Engagement der Gegner im Streit um den Fluglärm ableiten:

1. Je mehr die Konfliktgegner die Berichterstattung als feindlich wabrnehmen, desto stärker ist ibr kommunikatives Engagement. Dies gilt umso mehr, je mehr sie die vermeintlich feindliche Berichterstattung für wirkungsvoll halten.

Eine Bestätigung dieser Annahme könnte man als Hinweis auf korrigierende Handlungen deuten - als Versuche, die vermeintlich feindliche und einflussreiche Berichterstattung durch kommunikatives Engagement auszugleichen.

2. Je mehr sich die Konfliktgegner mit feindlichen Meinunsgklimata in Politik und Gesellschaft konfrontiert sehen, desto stärker ist ibr kommunikatives Engagement.

Betätigungen dieser Annahme könnte man ebenfalls als korrigierende Handlungen deuten - als Versuche, der vermeintlichen Skepsis in Politik und Gesellschaft durch kommunikatives Engagement entgegenzuwirken.

Die Wahrnehmung einer feindlichen Berichterstattung bezieht sich auf die Inhalte der Medien. Neben den wahrgenommenen Inhalten dürfte die wahrgenommene Offenheit der Medien für die eigenen Positionen ausschlaggebend für das Kommunikationsverhalten der Konfliktgegner sein. Man kann annehmen, dass sich die Konfliktgegner umso stärker kommunikativ engagieren, je weniger sie glauben, dass sie mit den eigenen Positionen bei den Medien durchdringen können.

3. Je weniger die Konfliktgegner eine Offenheit der Medien für die eigene Position wabrnehmen, desto stärker ist ibr kommunikatives Engagement.

Auch diesen Befund könnte man als Hinweise auf korrigierende Handlungen auffassen - Versuche, sich mit Leserreaktionen online und offline, mit Lobbying oder medienwirksamen Protestaktionen in Gesellschaft und Medien Gehör zu verschaffen.

Wie oben dargestellt wurde, handelt es sich bei den Konfliktgegnern im Streit um den Fluglärm um sehr unterschiedliche Gruppen von Akteuren, die vermutlich zum Teil in unterschiedlichem, zum Teil in ähnlichem Maße Insider- (Lobbying) und Außenseitertaktiken (Verbreitung von Informationen, Protestaktivitäten) anwenden. Die Relevanz der einzelnen Kommunikationsstrategien für die Konfliktparteien ist allerdings nicht bekannt. Daher soll die folgende Frage geklärt werden:

Welche Kommunikationsstrategien verfolgen die Gegner im Streit um den Fluglärm - welche Rolle spielen für sie Insider-(Lobbying, direkte Kontakte zur Politik) und Außenseiterstrategien (Proteste, Verbreitung von Informationen in der Öffentlichkeit)? 


\section{Methode}

\subsection{Befragung}

Grundlage der Untersuchung ist eine Online-Befragung der Konfliktgegner in der deutschen Fluglärmdebatte. Grundgesamtheit sind die Sprecher von Bürgerinitiativen auf der einen sowie Sprecher von Unternehmen und Verbänden der Luftverkehrswirtschaft und direkt abhängiger Industriezweige auf der anderen Seite. Die Fluglärmgegner wurden über das Webangebot der Bundesvereinigung gegen Fluglärm (BVF) identifiziert. Dort findet sich ein Verzeichnis der Sprecher aller Mitgliedsinitiativen in Deutschland. Diese Liste wurde ergänzt durch ein Verzeichnis deutscher Bürgerinitiativen gegen Fluglärm, das im Webangebot des Deutschen Fluglärmdienstes (DFLD) aufgeführt ist. Beide Listen waren in großen Teilen identisch und enthielten insgesamt 177 Fluglärminitiativen. Für jede wurde der Sprecher als Adressat der Befragung erfasst.

Die Befürworter des Luftverkehrs wurden in einem mehrstufigen Verfahren identifiziert. Im ersten Schritt wurden aus der „Lobby-Liste“ des Deutschen Bundestages alle relevanten Industrieverbände identifiziert. In der Lobby-Liste können sich Interessensverbände registrieren. Dies dürften fast alle Verbände tun, weil gemeinhin angenommen wird, dass das die Chancen auf Einladungen zu öffentlichen Anhörungen in den Bundestag erhöht (Deutsches Institut für Public Affairs 2006). In der Lobby-Liste fanden sich acht Verbände der Luftverkehrswirtschaft, deren Sprecher als Adressaten erfasst wurden. ${ }^{2}$ Im zweiten Schritt wurden alle Verbandsmitglieder in die Liste aufgenommen, die an der Entwicklung des Luftverkehrs in Deutschland beteiligt sind: Mitglieder, die den Luftverkehr betreiben (Airlines), Infrastruktur bereitstellen (Flughäfen) oder den Luftverkehr interessenspolitisch unterstützen (z. B. Unternehmensverbände, Industrieund Handelskammern). Im dritten Schritt wurden die Webangebote aller bisher identifizierten Adressaten nach Verbündeten in der Fluglärmdebatte durchsucht. Sie fanden sich häufig als Unterzeichner von Positionspapieren oder öffentlichen Stellungnahmen. Alle Partner, die explizit den Nachtflug oder allgemein die Ausweitung des Luftverkehrs fordern, wurden in die Liste der Adressaten aufgenommen. Durch dieses Verfahren wurden 149 Organisationen identifiziert, darunter Flughafenbetreiber, Airlines, Industrie- und Handelskammern, regionale und nationale Unternehmensverbände, Post- und Kurierdienste sowie Logistik- und Frachtunternehmen. Aus jeder Organisation wurde der Ansprechpartner für Fluglärm recherchiert. Wenn eine solche Person nicht existierte, wurde der Pressesprecher als Adressat der Befragung erfasst.

Der Fragebogen war vom 1. Juli bis zum 15. August 2015 im Feld. Die Adressaten erhielten per E-Mail eine Ankündigung mit einer kurzen Beschreibung des Untersuchungsinteresses an der Medienberichterstattung über die Fluglärmdebatte. Anschließend erhielten sie im Abstand von jeweils zehn Tagen eine Mail plus zwei Erinnerungsmails mit einem Link zum Online-Fragebogen. Pro Adressat konnte der Fragebogen nur einmal ausgefüllt werden. Zahlreiche Adressaten waren an der Befragung sehr interessiert. Viele füllten nicht nur den Fragebogen aus, sondern lieferten zusätzliche Einschätzungen per Telefon oder E-Mail. Andere waren schwieriger zur Teilnahme zu bewegen. Einige Sprecher in Unternehmen waren schwer per E-Mail zu erreichen. Einige Fluglärmgegner im Rhein-Main-Gebiet waren gegenüber der Studie sehr misstrauisch und konnten gar nicht oder erst nach Klärung einiger Fragen (z. B. zur Finanzierung der Studie) zur Teilnahme bewogen werden. Mehrere Fluglärminitiativen aus einer Stadt im

2 Unternehmensverbände der Luftfahrtindustrie, die die Interessen der technischen Hersteller von Luftfahrzeugen vertreten, wurden nicht berücksichtigt, weil sich ihnen der Fluglärm vor allem als technisches Problem stellt (z. B. als Anreiz zur Konstruktion leiserer Maschinen). 
Rhein-Main-Gebiet verweigerten kollektiv die Teilnahme. Sie begründeten ihre Entscheidung mit ihrer Sorge, von Vertretern der Luftverkehrswirtschaft ausgehorcht zu werden. Ein weiterer Teilnehmer erklärte diese Sorge: Für die Fluglärmgegner „sind wissenschaftliche Studien von ,unbekannten' Personen sehr suspekt. Allzu oft mussten sie erleben, wie unter dem Mantel der Wissenschaft mit höchst fragwürdigen Methoden, die allzu oft selbst grundlegendsten wissenschaftlichen Kriterien nicht genügten, Schindluder getrieben wurde."

Trotz der erwähnten Vorbehalte war die Teilnahmebereitschaft beider Konfliktparteien relativ hoch. Unter den 149 Organisationen, die die Ausweitung des Luftverkehrs befürworteten, gab es vier stichprobenneutrale Ausfälle. Es handelte sich um Organisationen, die sich mit dem Logistik-Standort Deutschland, nicht aber mit der Fluglärmdebatte auseinandersetzten. Von den restlichen 145 Organisationen füllten $48(33 \%)$ den Fragebogen aus. Die meisten Teilnehmer (50\%) sind Vertreter der Luftverkehrswirtschaft, einige arbeiten bei Verbänden (8\%), in der Beratung (8\%), für Logistikunternehmen oder Kurierdienste (6\%) oder in Industrie- und Handelskammern (6\%). Der Rest arbeitet in anderen Branchen (z. B. Tourismus) oder beantwortete die Frage nicht. Im Durchschnitt sind die teilnehmenden Befürworter des Luftverkehrs 49 Jahre alt. Die meisten (85 \%) haben einen Hochschulabschluss. 71 Prozent sind Männer, 19 Prozent sind Frauen. Der Rest hat die Frage nach dem Geschlecht nicht beantwortet.

Von den 177 Sprechern der Bürgerinitiativen hatte einer sein Engagement aus Gesundheitsgründen eingestellt. Von den übrigen 176 Sprechern nahmen 82 (47\%) an der Befragung teil. Der Rücklauf war in den verschiedenen Regionen aus den genannten Gründen unterschiedlich hoch. Die meisten Fluglärminitiativen befinden sich im RheinMain-Gebiet. Viele gibt es auch um die Flughäfen in München, Köln/Bonn, Berlin und Halle/Leipzig. Die Bürgerinitiativen um den Frankfurter Flughafen sind in der Befragung unterrepräsentiert. Der Rücklauf im Rhein-Main-Gebiet betrug nur 24 Prozent. In der Region um München betrug er 54 Prozent, in Köln/Bonn 47 Prozent, in Berlin 54 Prozent, in Halle/Leipzig 60 Prozent und in allen übrigen Regionen zusammengenommen 71 Prozent. Angesichts des Befragungsthemas - des deutschlandweiten Fluglärmstreits und der Medienberichterstattung - dürften die Unterschiede unproblematisch sein. Das Durchschnittsalter der Sprecher der Fluglärminitiativen ist mit 60 Jahren recht alt, das Bildungsniveau zudem hoch: Zwei Drittel haben einen Hochschulabschluss. Weitere 15 Prozent haben das Abitur. Nur 15 Prozent haben einen niedrigeren Bildungsabschluss. Das hohe Durchschnittsalter sowie das hohe Bildungsniveau könnten auf den Umstand verweisen, dass sich vor allem sesshafte Hausbesitzer, und hier vermutlich aus Zeitgründen vor allem Ruheständler als Sprecher von Fluglärminitiativen engagieren. Die weitaus meisten Teilnehmer sind Männer (73\%), nur 21 Prozent sind Frauen. Die restlichen Fluglärmgegner (6\%) haben die Frage nicht beantwortet.

\subsection{Fragebogen und Variablen}

Da es hier um Ansichten der Sprecher von Initiativen bzw. Organisationen ging, wurden die Sprecher die ganze Befragung hindurch immer wieder gebeten, aus Sicht ihrer Initiative bzw. Organisation zu antworten. Es ging bspw. um das, „was Sie oder andere Angehörige Ihres Unternehmens / Ihrer Organisation / Ihrer Bürgerinitiative so beobachten“ oder wodurch „Sie oder andere Vertreter Ihres Unternehmens / Ihrer Organisation / Ihrer Bürgerinitiative" aktiv geworden sind usw. 


\section{Abbängige Variablen}

Die Kommunikationsaktivitäten der Konfliktgegner zur Unterstützung ihrer Position wurden mit zwei Fragen ermittelt. Zunächst wurden sie gefragt: „Wie häufig haben Sie oder andere Vertreter Ihres Unternehmens / Ihrer Organisation / Ihrer Bürgerinitiative in den vergangenen zwei Jahren durch die folgenden Aktivitäten Unterstützung für ihre Position im Streit über den Luftverkehr gesucht?” Vorgelegt wurden insgesamt zehn Aktivitäten (z. B. „eine Demonstration organisiert”, „eine Unterschriftenaktion gestartet”, „einen Politiker kontaktiert”), die auf einer 7-stufigen Skala von „nie” (1) bis „sehr häufig” (7) einzuschätzen waren. Dann wurde gefragt: „Wie oft haben Sie durch eine der nachfolgenden Möglichkeiten zum Luftverkehr Stellung genommen?” Vorgelegt wurden insgesamt sechs Kommunikationsmöglichkeiten (z. B. „einen Leserbrief geschrieben”, „Newsletter oder Informationsmaterial verschickt”, „einen Onlineartikel kommentiert”), die ebenfalls auf einer 7-stufigen Skala von „nie” (1) bis „sehr häufig” (7) einzuschätzen waren.

Insgesamt 11 Items wurden in drei Skalen so zusammengefasst, dass sie nach den beschriebenen Außenseiter- und Insidertaktiken nach Kriesi (2001: 22f.) unterschieden werden können und für beide Gruppen reliabel sind. ${ }^{3}$ Bei den Taktiken der Insider handelt es sich wie erwähnt um die offizielle Lobbyarbeit der Insider sowie die „Protest-“ und „Informationspolitik“ der Außenseiter.

- Als offizielle Lobbyarbeit wurden die Items ,einen Politiker kontaktiert“, „ein Fachoder Hintergrundgespräch mit einem Politiker geführt“, „eine Stellungnahme herausgegeben“ sowie „mit Politikern gesprochen“ zusammengefasst. Die Skala erzielte in beiden Gruppen eine sehr hohe Reliabilität (Fluglärmgegner: Cronbach’s $\alpha=0,888$; Befürworter des Luftverkehrs: Cronbach's $\alpha=0,939)$.

- Zur Protestpolitik wurden die Items „zu einer Demonstration aufgerufen“, „an einer Demonstration teilgenommen “, ,eine Unterschriftenaktion gestartet“, ,, an einer Unterschriftenaktion teilgenommen" zusammengefasst. Auch hier sind die Reliabilitäten hoch (Fluglärmgegner: Cronbach's $\alpha=0,835$; Befürworter des Luftverkehrs: Cronbach's $\alpha=0,703$ ).

- Die strategische Verbreitung von Informationen bezieht sich hier konkret auf das Verfassen von Leserkommentaren online und offline. Hierzu wurden die Items „einen Leserbrief geschrieben“, „einen Online-Artikel kommentiert“, „in einem Diskussionsforum geschrieben " zusammengefasst. Auch hier sind die Reliabilitätswerte in beiden Gruppen hoch (Fluglärmgegner: Cronbach's $\alpha=0,751$; Befürworter des Luftverkehrs: Cronbach's $\alpha=0,794)$.

Damit die Werte der Skalen miteinander verglichen werden können, wurden sie jeweils durch die Anzahl ihrer Items geteilt. Auf diese Weise erhält jede Skala einen Wertbereich zwischen [1; 7]. Die Mittelwerte werden im Ergebnisteil verglichen.

3 Die Skalen wurden auf der Grundlage explorativer, getrennt für die beiden Befragtengruppen berechneter Faktorenanalysen gebildet. Zunächst wurden zum Zwecke der Vergleichbarkeit der beiden Gruppen fünf Items aus der Analyse ausgeschlossen, die in beiden Gruppen auf unterschiedlichen Faktoren luden. Eine explorative Faktorenanalyse der übrigen Items ergab für beide Gruppen die beschriebenen drei Faktoren, die weitgehend den Kommunikationstaktiken nach Kriesi (2001) entsprechen. Bei den Befürwortern des Luftverkehrs waren die Items „eine Unterschriftenaktion starten“ und „an einer Unterschriftenaktion teilnehmen“ nicht trennscharf zum Faktor „Leserkommentare“. Aus theoretischen Gründen und aufgrund des guten Reliabiltätswertes wurden beide Items dennoch zum Faktor „Protestaktionen“ zusammengefasst. 


\section{Unabhängige Variablen}

Wabrnebmung einer feindlichen Berichterstattung: Die Teilnehmer wurden gefragt: „Verschiedene Medien berichten natürlich ganz unterschiedlich. Was fällt Ihnen an der Berichterstattung über den Streit um den Luftverkehr am meisten auf: Berichten die Medien neutral, zugunsten der Reduzierung oder zugunsten der Ausweitung des Luftverkehrs?" Die Teilnehmer sollten das auf einer sieben-stufigen Skala von „eindeutig zugunsten der Reduzierung“ (1) bis „eindeutig zugunsten der Ausweitung“ (7) einschätzen. Die Angehörigen beider Konfliktparteien nehmen die Berichterstattung deutlich als gegen ihre eigene Position gerichtet wahr. Die Fluglärmgegner glauben, dass die Medien zugunsten einer Ausweitung des Luftverkehrs berichten ( $\mathrm{M}=5.24, \mathrm{SD}=1.238)$, während die Befürworter glauben, dass die Medien zugunsten einer Reduzierung des Luftverkehrs berichten $(\mathrm{M}=2.42, \mathrm{SD}=964)$. Der Unterschied ist hoch signifikant $(\mathrm{T}=$ $14.440, \mathrm{df}=17.544, \mathrm{p}=.000$ ). Für die folgende Analyse wurde die Wahrnehmung einer feindlichen Berichterstattung für beide Konfliktparteien so recodiert, dass hohe Werte für die Wahrnehmung einer starken Medienfeindlichkeit, niedrige Werte für die Wahrnehmung einer starken Medienfreundlichkeit stehen.

Vermutete Medienwirkungen: Die Befragten sollten auf einer sieben-stufigen Skala einschätzen, wie „schwach“ (1) oder „stark“ (7) die Berichterstattung über den Luftverkehr nach ihrer Beobachtung „Politiker“ und die „deutsche Öffentlichkeit“ beeinflusst. Die Befürworter des Luftverkehrs schätzen den Medieneinfluss auf Politiker $(\bar{x}=4,88$, $\mathrm{SD}=1,632)$ sowie auf die deutsche Öffentlichkeit $(\bar{x}=4,68, \mathrm{SD}=1,337)$ deutlich stärker ein als die Fluglärmgegner (Einfluss auf Politiker: $\bar{x}=3,21$; $\mathrm{SD}=2,017$; Einfluss auf Öffentlichkeit: $\bar{x}=3,54, \mathrm{SD}=1,557)$. Die Unterschiede sind hoch signifikant $(\mathrm{p}=0,000$ nach Mann-Whitney-U-Test).

Interaktion zwischen vermuteten Medienwirkungen und der Wabrnebmung feindlich verzerrter Berichterstattung: Einige Studien haben gezeigt, dass die Wahrnehmung einer feindlichen Berichterstattung umso eher mit dem Verhalten der Anhänger von Konfliktlagern zusammenhängt, je mehr Wirkung sie der Berichterstattung zuschreiben. Daher wurde die Wahrnehmung der Medieninhalte durch die Konfliktgegner mit ihren Einschätzungen der Medienwirkungen auf „Politiker“ und die „deutsche Öffentlichkeit" gewichtet. Zur Berechnung der Interaktionsterme wurden die Variablen zentriert, d. h. für jede Variable wurde von den fallbezogenen Werten der jeweilige Mittelwert abgezogen. Hierdurch erhielt jede Variable einen neuen Mittelwert von $\bar{x}=0$. Zentrierung wird empfohlen, um Kollinearität zwischen dem Interaktionsterm und den ungewichteten Variablen zu vermeiden (Richter 2007).

Wabrnehmung des Meinungsklimas: Die Wahrnehmung der Meinungsklimata in verschiedenen sozialen Bereichen wurde in einer Frage ermittelt: „Was vermuten Sie: Wie sehen das [ob der Luftverkehr in Deutschland reduziert oder ausgeweitet werden soll] die folgenden Akteure?" Einschätzen sollten die Befragten u. a. die Sichtweisen „der meisten Deutschen“ und "der meisten Politiker“. Beide Konfliktparteien haben eine ähnliche Vorstellung von den Sichtweisen der „meisten Deutschen“. Im Durchschnitt glauben sie, dass die meisten Deutschen weder eine Reduzierung noch eine Ausweitung des Luftverkehrs befürworten (Fluglärmgegner: $\bar{x}=3,65, \mathrm{SD}=1,047$; Befürworter: $\bar{x}=$ $3,72, \mathrm{SD}=1,330 ; \mathrm{T}=-0,341, \mathrm{df}=78.804$, n.s.). Die Kontrahenten schätzen aber die Sichtweisen von Politikern signifikant unterschiedlich ein. Aus Sicht der Fluglärmgegner sind die meisten Politiker für die Ausweitung des Luftverkehrs. Aus Sicht der Befürworter sind die meisten Politiker weder für die Reduzierung noch für die Ausweitung (Fluglärmgegner: $\bar{x}=5,57, \mathrm{SD}=1,111$; Befürworter: $\bar{x}=3,98, \mathrm{SD}=1,113 ; \mathrm{T}=7,817$, $\mathrm{df}=127, \mathrm{p}=0,000)$. 
Medien-Wirksamkeit: Die Teilnehmer wurden gefragt: „Wie gut können Sie Ihre Position im Streit um den Luftverkehr in die Medienberichterstattung einbringen?“ Einzuschätzen war auf einer 7-stufigen Skala von „trifft gar nicht zu“ (1) bis „trifft voll

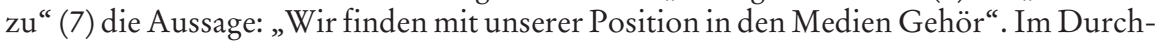
schnitt lehnten beide Konfliktparteien diese Aussage weder ab, noch stimmten sie ihr zu. Einen signifikanten Unterschied zwischen den Einschätzungen beider Konfliktparteien gibt es nicht (Fluglärmgegner: $\bar{x}=3,85, \mathrm{SD}=1,754$; Befürworter des Luftverkehrs: $\bar{x}=3,94, \mathrm{SD}=1,420 ; \mathrm{T}=-0,296, \mathrm{df}=112.702$, n.s.).

\section{Kontrollvariablen}

Als Kontrollvariable wird neben Alter, Bildungsgrad die eigene Position der Befragten in der Debatte um den Luftverkehr berücksichtigt. Der angegebene Bildungsabschluss wurde in Bildungsjahre umgerechnet. Ihre eigene Position schätzten die Teilnehmer auf einer 7-stufigen Skala von „der Luftverkehr in Deutschland sollte erheblich reduziert“ (1) bis „sollte erheblich ausgeweitet werden“ (7). Im Durchschnitt meinen die Fluglärmgegner, der Luftverkehr solle deutlich reduziert werden $(\bar{x}=1,95, \mathrm{SD}=1,115)$. Dagegen meinen die Befürworter, der Luftverkehr solle eher ausgeweitet werden $(\bar{x}=5,20$, $\mathrm{SD}=9,919)$. Ihre Einschätzungen unterscheiden sich signifikant $(\mathrm{T}=-17,444 ; \mathrm{df}=121$; $\mathrm{p}=0,000)$.

\section{Ergebnisse}

\subsection{Kommunikationsstrategien}

Soweit es um die hier abgefragten Aktivitäten geht, lassen sich bei den Konfliktparteien unterschiedliche Kommunikationstaktiken feststellen. Die Fluglärmgegner und die Befürworter des Luftverkehrs engagieren sich im Durchschnitt ähnlich häufig durch Lobby-Arbeit, d. h. durch direkte Kontakte zur Politik und Herausgabe von Stellungnahmen (Fluglärmgegner: $\bar{x}=4,98, \mathrm{SD}=1,40$; Befürworter des Luftverkehrs: $\bar{x} 4,52$; SD $=1,68$, n.s. nach Mann-Withney-U-Test $\left.{ }^{4}\right)$. Die beiden Konfliktparteien vertreten ihre Positionen aber unterschiedlich häufig durch die Teilnahme an Protestaktivitäten und Leserkommentare. Die Fluglärmgegner engagieren sich im Durchschnitt weitaus häufiger als die Befürworter des Luftverkehrs durch Protest- und Unterschriftenaktionen (Fluglärmgegner: $\bar{x}=4,79 ; \mathrm{SD}=1,61$; Befürworter des Luftverkehrs: $\bar{x}=1,44 ; \mathrm{SD}=0,75)$ sowie durch Leserkommentare (Fluglärmgegner: $\bar{x}=4,16$; $\mathrm{SD}=1,63$; Befürworter des Luftverkehrs: $\bar{x}=1,74 ; \mathrm{SD}=1,11)$. Die Unterschiede zwischen den Konfliktparteien sind in beiden Fällen hochsignifikant ( $p=0,001$, Mann-Withney-U-Test). Dies kann man als Hinweise darauf interpretieren, dass die drei untersuchten Kommunikationsstrategien - Lobby-Arbeit, Engagement in Protest- und Unterschriftenaktionen sowie Informationspolitik durch Leserkommentare - für die Fluglärmgegner gleichermaßen wichtig sind. Für die Befürworter des Luftverkehrs ist dagegen vor allem die Lobby-Arbeit wichtig. Protestteilnahmen und Leserkommentare spielen für sie fast keine Rolle. Allerdings kann man diese Feststellung nur auf der Grundlage der hier abgefragten Aktivitäten machen. Sie sind daher mit Vorsicht zu interpretieren. Es kann nicht ausgeschlossen werden, dass die Konfliktparteien Lobby-, Protest- oder Informationsaktivitäten betreiben, die hier nicht abgefragt wurden.

4 Da nicht alle Variablen in den beiden Gruppen normalverteilt sind, liegen den Signifikanztests nicht-parametrische Mann-Withney-U-Tests für unabhängige Stichproben zugrunde. 


\subsection{Einflüsse auf das Kommunikationsverbalten}

Der Einfluss der Wahrnehmungen der Berichterstattung, der Vermutungen von Medienwirkungen und der Wahrnehmungen des Meinungsklimas auf die Kommunikationsstrategien wird mit Regressionsanalysen geprüft. Aufgrund der geringen Fallzahlen und der daraus resultierenden Schwierigkeit, identische aussagekräftige Modelle zu entwickeln, wurden schrittweise Regressionsanalysen durchgeführt. ${ }^{5}$ Sie zeigen, dass sich die Fluglärmgegner durch Proteste und Unterschriftenaktionen, durch Kontakte in die Politik und Leserkommentare unabhängig davon engagieren, ob sie die Berichterstattung als feindlich wahrnehmen. Ihre Wahrnehmung der Berichterstattung hat keinen Einfluss auf die Intensität, mit der sie die einzelnen Kommunikationsstrategien verfolgen. Dies widerspricht der Annahme, dass die Fluglärmgegner auf die vermeintlich feindliche Berichterstattung mit korrigierenden Handlungen reagieren - mit Aktivitäten, die die Berichterstattung ausgleichen sollen.

Die Befürworter des Luftverkehrs engagieren sich dagegen zum Teil durchaus in Abhängigkeit ihrer Wahrnehmung der Berichterstattung: Je mehr sie die Berichterstattung als feindlich wahrnehmen, desto häufiger betreiben sie Lobby-Arbeit. Je mehr sie die Berichterstattung zudem gegen sich wahrnehmen und zugleich vermuten, dass die Medieninhalte die Öffentlichkeit beeinflussen, desto häufiger verfassen sie Leserbriefe oder Onlinekommentare. Dies sind deutliche Hinweise auf korrigierende Handlungen: Es erscheint durchaus plausibel, dass die Befürworter des Luftverkehrs versuchen, die vermeintlich feindliche Berichterstattung durch Lobbying und Leserkommentare auszugleichen. Dagegen scheinen Protestaktivitäten wie Demonstrationen oder Unterschriftenaktionen für sie kein adäquates Mittel, um der Berichterstattung entgegenzuwirken: Hier zeigt sich kein Zusammenhang zwischen der Wahrnehmung einer feindlichen Berichterstattung und der Intensität ihres Engagements.

Als korrigierend kann man das kommunikative Engagement der Gegner in Konflikten auch dann deuten, wenn es eine Reaktion auf die Wahrnehmung eines feindlichen Meinungsklimas ist. Der Zweck des Engagements liegt dann nicht darin, ein Gegengewicht zur vermeintlich negativ verzerrten Berichterstattung, sondern zur wahrgenommenen Skepsis in der Öffentlichkeit oder in relevanten Personenkreisen herzustellen. Bei den Fluglärmgegnern deutet sich das in nur einem der geprüften Zusammenhänge an: Je mehr die Fluglärmgegner glauben, dass „die meisten Deutschen“ gegen sie sind, desto häufiger verfassen sie Leserkommentare. Allerdings zeigt sich kein solcher $\mathrm{Zu}$ sammenhang, wenn es um Verbindungen zu Politikern oder um Protestaktivitäten geht. Bei den Befürwortern des Luftverkehrs wirkt die Wahrnehmung des Meinungsklimas in die entgegengesetzte Richtung: Die Befürworter betreiben umso intensivere LobbyArbeit, je mehr sie „die meisten Deutschen“ in der Fluglärmdebatte auf ihrer Seite glau-

5 In schrittweisen Regressionsanalysen werden nicht alle unabhängigen Variablen gleichzeitig in die Regressionsmodelle eingeschlossen, sondern nach einem Algorithmus sukzessiv ausgewählt: Zunächst wird eine einfache Regression mit derjenigen unabhängigen Variable durchgeführt, die am stärksten mit der abhängigen Variable korreliert. In den nächsten Schritten werden die unabhängigen Variablen in die Regression eingeschlossen, die den größten partiellen Korrelationskoeffizienten aufweisen. Auf diese Weise werden die relevantesten Einflussvariablen identifiziert. Unabhängige Variablen wurden nur dann in die Regression eingeschlossen, wenn ihr partieller Korrelationskoeffizient das Signifikanzniveau von 0,09 nicht unterschreitet. Umgekehrt wurden alle Variablen aus dem Modell entfernt, deren partieller Korrelationskoeffizient das Signifikanzniveau von 0,09 überschreitet (zur schrittweisen Regression s. Backhaus et al. 2011: 107-112). 


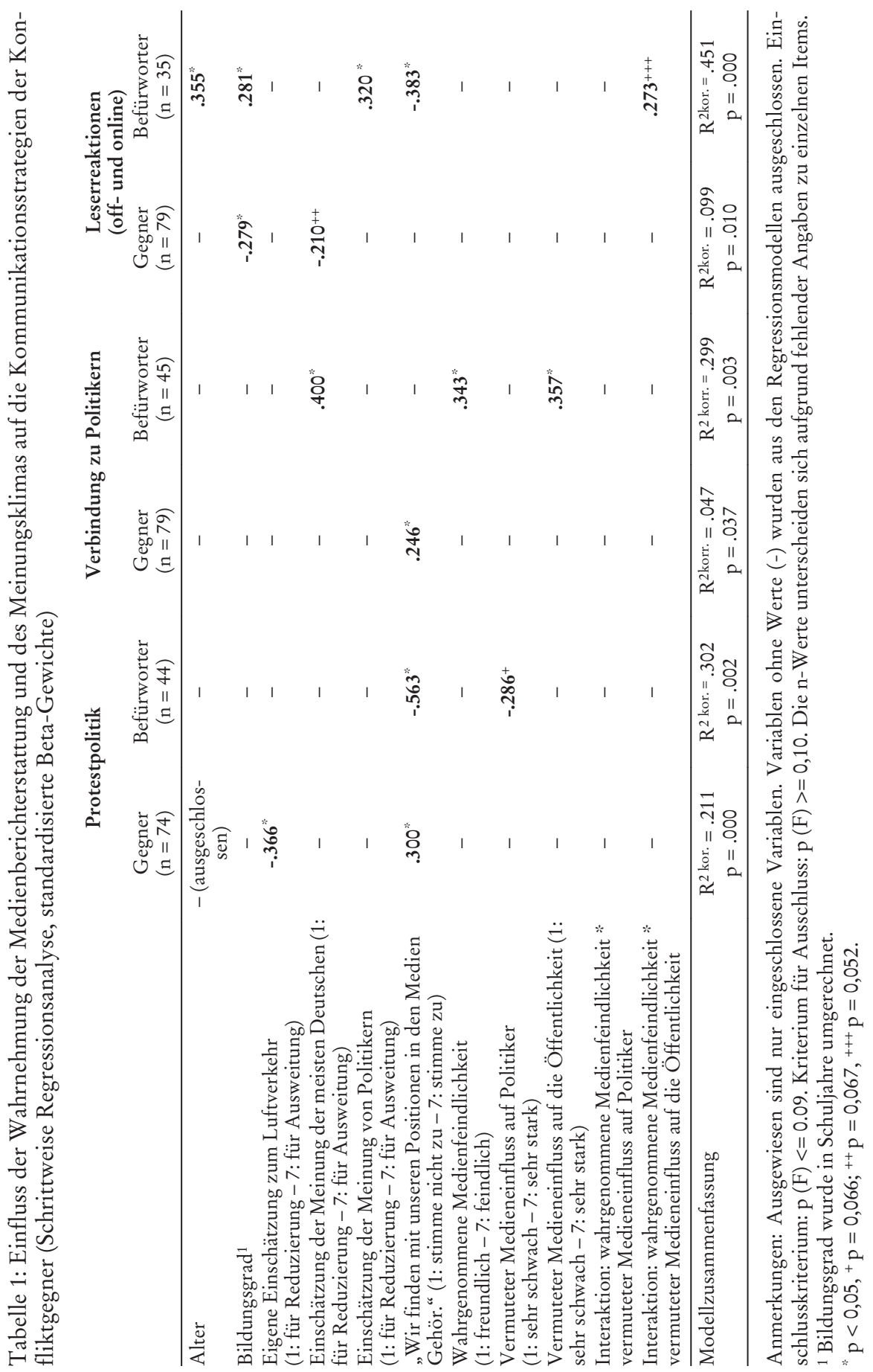


ben. Und sie verfassen umso häufiger Leserreaktionen, je mehr sie Politiker auf ihrer Seite glauben. Dies widerspricht der Hypothese der korrigierenden Handlungen. Die Befürworter des Luftverkehrs intensivieren ihre offizielle Kommunikation bzw. ihre Leserreaktionen vermutlich nicht, um sich gegen vermeintlich feindliche Meinungsklimata in Gesellschaft bzw. Politik zu stellen. Möglicherweise nutzen sie diese Kommunikationswege im Gegenteil vielmehr dann, wenn sie sich Rückenwind aus Gesellschaft und Politik zunutze machen können.

Während es bei der Wahrnehmung einer feindlichen Berichterstattung um den inhaltlichen Aspekt der Berichterstattung geht, geht es bei der Medienwirksamkeit um den Eindruck, die Berichterstattung zugunsten der eigenen Positionen beeinflussen zu können. Als Hinweis auf korrigierende Handlungen kann man das Kommunikationsengagement von Akteuren dann interpretieren, wenn sie aus ihrem Eindruck resultieren, dass sie in den Medien mit ihren Positionen nicht durchdringen können. Ein verstärktes Kommunikationsengagement hat dann vermutlich den Zweck, den eigenen Positionen auf alternativen Wegen oder durch medienwirksame Inszenierungen Gehör zu verschaffen. Bei den Fluglärmgegnern finden sich solche Zusammenhänge nicht. Im Gegenteil: Die Fluglärmgegner betreiben Protest- und Unterschriftenaktionen sowie Lobby-Arbeit umso intensiver, je mehr sie den Eindruck haben, dass sie mit ihren Positionen in den Medien Gehör finden können. Die Fluglärmgegner sind zu Protesten und LobbyArbeit möglicherweise vor allem dann bereit, wenn sie den Eindruck haben, dass sie die Berichterstattung zu ihren Gunsten beeinflussen können. Bei den Befürwortern des Luftverkehrs finden sich umgekehrte Zusammenhänge und damit deutliche Hinweise auf korrigierende Handlungen. Die Befürworter engagieren sich durch Protestaktivitäten wie Unterschriftenaktionen und Demonstrationen sowie Leserreaktionen umso intensiver, je mehr sie den Eindruck haben, dass sie mit ihren Positionen in den Medien kein Gehör finden. Möglicherweise versuchen sie, dem mangelnden Medieninteresse durch intensivierte Kommunikationsanstrengungen entgegenzuwirken.

Neben den beschriebenen Faktoren beeinflussen die eigene Einstellung sowie Alter und Bildung einzelne Kommunikationstaktiken. So mobilisieren sich die Fluglärmgegner umso häufiger zu Protestaktivitäten, je mehr sie wollen, dass der Luftverkehr in Deutschland reduziert wird. Auf die anderen Kommunikationstaktiken der Fluglärmgegner sowie die Kommunikationstaktiken der Befürworter des Luftverkehrs hat die eigene Einstellung dagegen keinen Einfluss. In beiden Konfliktparteien hängt die Bereitschaft, Leserkommentare zu verfassen, vom Bildungsgrad ab. Allerdings zeigen sich hier gegensätzliche Effekte: Die Fluglärmgegner verfassen umso seltener, die Befürworter umso häufiger Leserkommentare, je höher sie gebildet sind. Bei den Befürwortern steigt die Bereitschaft, Leserkommentare zu verfassen, zudem mit dem Alter. Vermutlich ist das Verfassen von Leserkommentaren in den Organisationen der Befürworter des Luftverkehrs sozusagen „Chefsache“ - also Teil der professionellen Kommunikationsstrategie der älteren und höher gebildeten Führungspersonen.

\section{Zusammenfassung und Diskussion}

Die Hypothesen der Untersuchung - dass die Antagonisten sich vor allem dann kommunikativ engagieren, wenn sie sich mit einer feindlichen Berichterstattung, feindlichen Meinungsklimata in Politik und Gesellschaft sowie mit schwer zu beeinflussenden Medien konfrontiert sehen - bestätigen sich für die Befürworter des Luftverkehrs, nicht aber für die Fluglärmgegner. Die Fluglärmgegner engagieren sich durch Protestaktivitäten und Kontakte zu Politikern umso intensiver, je mehr sie den Eindruck haben, dass sie mit ihren Positionen in den Medien Gehör finden können. Sie engagieren sich also 
nicht wie vermutet dann, wenn sie bei den Medien ein mangelndes Interesse an ihren Positionen wahrnehmen, sondern vielmehr dann, wenn sie Offenheit wahrnehmen. Ihr Engagement setzen sie folglich vermutlich eher dazu ein, Rückenwind aus den Medien zu nutzen als dazu, sich bei Gegenwind zu behaupten. Einzig wenn es um Leserkommentare geht, finden sich Hinweise auf korrigierende Handlungen: Die Fluglärmgegner verfassen umso häufiger Leserkommentare, je mehr Skepsis sie in der Öffentlichkeit wahrnehmen. Möglicherweise nutzen sie solche Informationskanäle, um der wahrgenommenen Skepsis in der Bevölkerung entgegenzuwirken.

Bei den Befürwortern des Luftverkehrs finden sich dagegen deutliche Hinweise auf korrigierende Handlungen. Die Befürworter intensivieren ihre Kontakte in die Politik und ihr Engagement durch Leserkommentare umso stärker, je mehr sie die Berichterstattung für feindlich und - im Falle ihres Engagements durch Leserkommentare - je mehr sie die vermeintlich feindliche Berichterstattung zudem für wirkungsvoll halten. Die Befürworter engagieren sich durch Protestaktivitäten und Leserkommentare zudem umso intensiver, je weniger sie den Eindruck haben, dass sie mit ihren Positionen in den Medien Gehör finden. Diese Befunde lassen die Vermutung zu, dass die Befürworter ihr Engagement als korrigierende Handlungen einsetzen, also dazu, die vermeintlich negative Berichterstattung auszugleichen bzw. dem geringen Medieninteresse entgegenzuwirken. Dabei handeln die Befürworter des Luftverkehrs nicht gänzlich unabhängig von den Meinungsklimata in Politik und Öffentlichkeit. Ihre Kontakte in die Politik sind umso intensiver, je mehr Unterstützung sie in der Öffentlichkeit wahrnehmen, und ihre Leserkommentare umso häufiger, je mehr Unterstützung unter Politikern sie wahrnehmen.

Die unterschiedlichen Zusammenhänge sind vermutlich auf Unterschiede zwischen den beiden Konfliktparteien zurückzuführen. Bei den Fluglärmgegnern handelt es sich um einen Verbund von Bürgerinitiativen. Ihr direkter Kontakt zur Politik hängt stark von ihrer öffentlichen Sichtbarkeit und davon ab, wie viel öffentlichen Druck auf die Politik sie aufbauen können. Das Fluglärmproblem existiert für Politik und Gesellschaft nur so lange, wie die Fluglärmgegner auf das Problem aufmerksam machen. Ihre Abhängigkeit von den Medien dürfte ein Grund dafür sein, dass sich die Fluglärmgegner vor allem dann öffentlich engagieren, wenn sie glauben, dass sie mit ihren Botschaften auf Medieninteresse stoßen. Bei den Befürwortern des Luftverkehrs handelt es sich dagegen um etablierte Interessen, die nicht nur aufgrund des Fluglärms, sondern aus vielen anderen Gründen - zum Teil auch in Konkurrenz zueinander - in Verbindung zur Politik stehen. Im Streit um den Fluglärm sind sie zudem vermutlich in einer reaktiven Position: Die Befürworter des Luftverkehrs würden den Fluglärm vermutlich nicht eigeninitiativ thematisieren, sondern dürften in erster Linie auf die Proteste der Fluglärmgegner reagieren. Dies dürfte ein Grund dafür sein, dass sie sich vor allem dann kommunikativ engagieren, wenn sie sich mit einer negativen Berichterstattung oder mit voreingenommenen Journalisten konfrontiert sehen und sich in der Öffentlichkeit dagegen behaupten wollen.

Ein weiterer wichtiger Unterschied zwischen den Konfliktparteien ist ihre Betroffenheit. Die Fluglärmgegner sind als Privatpersonen vom Fluglärm betroffen. Die Befürworter des Luftverkehrs werden als Berufstätige mit dem Fluglärmstreit konfrontiert. Dies dürfte erklären, warum sich die Fluglärmgegner, aber nicht die Befürworter in Abhängigkeit ihrer eigenen Position im Fluglärmstreit engagieren: Die Fluglärmgegner engagieren sich umso häufiger in Protesten oder Unterschriftenaktionen, je mehr sie den Luftverkehr in Deutschland reduziert sehen wollen. Die Befürworter des Luftverkehrs engagieren sich dagegen unabhängig von ihrer eigenen Position. 
In zukünftigen Studien müssten weitere Variablen als Kontrolle für die Unterschiede zwischen den Konfliktparteien eingeschlossen werden, die hier nicht berücksichtigt wurden, bspw. die Identifikation der Antagonisten mit der eigenen Konfliktpartei (Hartmann \& Tanis 2013; Reid 2012; Stroud et al. 2014), der wahrgenommene gesellschaftliche Status der eigenen Konfliktpartei (Hartmann \& Tanis 2013), die Homogenität der Konfliktparteien und die Größe der eigenen Organisation.

Befragt wurden in dieser Untersuchung die Sprecher von Organisationen oder Bürgerinitiativen. Dies hat Vor- und Nachteile: Ein Nachteil ist, dass hier keine Aussagen über die zahlreichen Individuen gemacht werden können, die als Angehörige oder Sympathisanten der Konfliktlager im Fluglärmstreit kommunizieren - bspw. in sozialen Netzwerken, als Blogger, mit Bannern auf Demonstrationen, mit Aufklebern auf Autos usw. Obwohl die Kommunikationsweisen dieser Personen relevant sind, fallen sie nicht in den Gegenstandsbereich dieser Studie. Gegenstand dieser Untersuchung sind die führenden Akteure im Fluglärmstreit - diejenigen auf beiden Seiten, die die jeweilige Konfliktpartei nach außen vertreten, ihre Kommunikationsstrategien festlegen, Verhandlungspositionen ausarbeiten und Aktivitäten koordinieren. Es handelt sich um diejenigen Personen, die als Vertreter von Initiativen oder Organisationen strategisch kommunizieren, ihre Mitglieder mobilisieren, als offizielle Medienquellen fungieren und daher die Konfliktdynamik maßgeblich beeinflussen.

Das Ziel dieser Studie war es, in einer ersten Annäherung reziproke Effekte der Konfliktberichterstattung auf die Antagonisten zu ermitteln. Es deutet sich an, dass die Konfliktberichterstattung von den Antagonisten auf beiden Seiten als feindlich wahrgenommen wird und dass diese Wahrnehmung sie zu unterschiedlichen Reaktionen veranlasst. Die Fluglärmgegner engagieren sich unabhängig von ihrer Wahrnehmung der Medieninhalte. Entscheidend ist für sie vielmehr der Eindruck, dass sie mit ihren Positionen Gehör finden können. Dagegen reagieren die Befürworter auf die vermeintlich feindliche Berichterstattung sowie auf ihren Eindruck, dass sie mit ihren Positionen bei den Medien nicht durchdringen mit einer Intensivierung ihres Engagements. Die Relevanz dieser Einflüsse liegt darin, dass die Reaktionen der Antagonisten weitere Folgen nach sich ziehen können, die die Konfliktdynamik entscheidend beeinflussen - bspw. Gegenreaktionen der gegnerischen Partei, Reaktionen von Journalisten, Politikern oder der Bevölkerung. Diese Prozesse sollten in nachfolgenden Studien genauer untersucht werden.

Die reziproken Effekte der Konfliktberichterstattung auf die Antagonisten wurden hier in einer ersten Annäherung untersucht, die viele Fragen offen lässt und Einschränkungen hat: Ein Problem ist die Kausalität. Auf der Grundlage einer Querschnittsbefragung lassen sich keine Kausalaussagen machen. Zwar erscheint es plausibel, und es bestätigt vorangegangene Untersuchungen, dass die Berichterstattung eine Ursache der strategischen Kommunikation der Konfliktparteien ist (z. B. Hwang et al. 2008). Der Zusammenhang könnte dennoch umgekehrt sein: die strategischen Kommunikationsaktivitäten und ihre Evaluation könnten eine Ursache der Bewertung der Berichterstattung und der Offenheit der Medien für die eigenen Positionen sein. Inwieweit die eine oder andere Interpretation hier zutrifft, lässt sich auf Basis dieser Querschnittsbefragung nicht klären. Vermutlich handelt es sich um prozesshafte Wechselwirkungen. Um die Dynamik von Konflikten und die treibenden Ursachen der Kommunikationsaktivitäten besser zu klären, wären daher in Zukunft Längsschnittuntersuchungen wünschenswert.

Eine weitere Einschränkung ergibt sich aus den geringen Fallzahlen: sie führen zu dem Risiko, dass relevante Zusammenhänge übersehen werden, weil sie keine statistische Signifikanz erreichen. Dies könnte ein Grund dafür sein, dass hier einige Zusammenhänge nur vereinzelt auftreten. Ein Beispiel ist die Frage, warum die Lobby-Arbeit der 
Befürworter mit ihrer Wahrnehmung einer feindlichen Berichterstattung zusammenhängt, ihr Engagement in Leserkommentaren hingegen mit ihrer Wahrnehmung einer feindlichen und zugleich wirkungsvollen Berichterstattung. Aufgrund der geringen Fallzahlen ist es möglich, dass hier weitere empirisch bedeutsame Zusammenhänge oder Muster übersehen wurden. Die Befunde können daher nur mit Vorsicht interpretiert und sollten in nachfolgenden, ähnlich gelagerten Fallstudien erhärtet werden.

Auf der Grundlage dieser Studie kann man auch deshalb nur mit Einschränkungen Aussagen über die reziproken Effekte in Konflikten machen, weil es sich hier um eine Fallstudie handelt. In Zukunft wären fallübergreifende Untersuchungen von Konflikten wünschenswert, um generelle Zusammenhänge zu identifizieren. Hierbei sollten die erwähnten Variablen berücksichtigt werden: Homogenität der Gruppe, Identifikation mit der eigenen Konfliktpartei, wahrgenommener Status der Konfliktpartei durch die Mitglieder usw. Zudem sollten neben der Intensität ihres Engagements auch andere Reaktionen der Antagonisten betrachtet werden - bspw. ihr Interesse an einem Kompromiss, ihre Dialogbereitschaft oder ihre Bereitschaft, Sachverhalte unsachgemäß - z. B. übertrieben oder unvollständig - darzustellen. Durch die vorliegende Studie wurden weitere wichtige Variablen identifiziert, die für solche Studien relevant wären: Wichtig wäre bspw. die Medienwirksamkeit als unabhängige oder moderierende Variable. Bei der Frage nach dem Engagement müsste außerdem sichergestellt sein, dass die vorrangigen Aktivitäten beider Konfliktparteien gleichermaßen berücksichtigt werden (z. B. Protest auf der einen, Lobby-Arbeit auf der anderen Seite).

\section{Literaturverzeichnis}

Backhaus, K., Erichson, B., Plinke, W., Weiber, R. (2011). Multivariate Analysemethoden. Eine anwendungsorientierte Einführung. Berlin: Springer.

Barnidge, M., Rojas, H (2014). Hostile Media Perceptions, Presumed Media Influence, and Political Talk: Expanding the Corrective Action Hypothesis. International Journal of Public Opinion Research 26(2), 135-56.

Bernhard, U.; Dohle, M. (2013). Indirekte Medienwirkungen bei der Volksabstimmung über „Stuttgart 21“. Wahrgenommene Medieneinflüsse und ihre Konsequenzen. Medien E Kommunikationswissenschaft 61(1), 38-57.

Bernhard, U. \& Dohle, M. (2015). Corrective or Confirmative Actions? Political Online Participation as a Consequence of Presumed Media Influences in Election Campaigns. Journal of Information Technology E Politics 12(3), 285-302.

Choi, J., Yang, M., Chang, J. J. (2009). Elaboration of the Hostile Media Phenomenon: The Roles of Involvement, Media Skepticism, Congruency of Perceived Media Influence, and Perceived Opinion Climate. Communication Research 36(1), 54-75.

Coe, K., Tewksbury, D., Bond, B. J., Drogos, K. L., Porter, R. W., Yahn, A., Zhang, Y. (2008). Hostile News: Partisan Use and Perceptions of Cable News Programming. Journal of Communication 58(2), 201-19.

Deutsches Institut für Public Affairs (2006). Verbandslobbying und Verbandsmanagement. Einfluss und Erfolgsfaktoren in Berlin und Brüssel. Münster [u.a.]: LIT.

Feldman, L., Hart, P. S., Leiserowitz, A., Maibach, E., Roser-Renouf, C. (2015). Do Hostile Media Perceptions Lead to Action? The Role of Hostile Media Perceptions, Political Efficacy, and Ideology in Predicting Climate Change Activism. Communication Research (online first, January 6,2015$)$.

Gunther, A. C. (1998). The Persuasive Press Inference Effects of Mass Media on Perceived Public Opinion. Communication Research, 25(5), 486-504.

Gunther, A. C., \& Schmitt, K. (2004). Mapping Boundaries of the Hostile Media Effect. Journal of Communication, 54(1), 55-70. 
Gunther, A. C., \& Liebhart, J. L. (2006). Broad Reach or Biased Source? Decomposing the Hostile Media Effect. Journal of Communication, 56(3), 449-466.

Gunther, A. C., Miller, N., \& Liebhart, J. L. (2009). Assimilation and Contrast in a Test of the Hostile Media Effect. Communication Research 36(6), 747-764.

Hartmann, T. \& Tanis, M. (2013). Examining the Hostile Media Effect as an Intergroup Phenomenon: The Role of Ingroup Identification and Status. In: Journal of Communication 63(3), 535-55.

Ho, S. S., Binder, A. R., Becker, A. B., Moy, P., Scheufele, D. A., Brossard, D., Gunther, A. C. (2011). The Role of Perceptions of Media Bias in General and Issue-Specific Political Participation. Mass Communication and Society 14(3), 343-74.

Huge, M., Glynn, C. J. (2010). Hostile Media and the Campaign Trail: Perceived Media Bias in the Race for Governor. Journal of Communication 60(1), 165-81.

Hwang, H., Pan, Z., Sun, Y. (2008). Influence of Hostile Media Perception on Willingness to Engage in Discursive Activities: An Examination of Mediating Role of Media Indignation. Media Psychology 11(1), 76-97.

Kepplinger, M. H. (2007). Reciprocal Effects: Toward a Theory of Mass Media Effects on Decision Makers. The Harvard International Journal of Press/Politics 12(2), 3-23.

Kepplinger, H. M., \& Zerback, T. (2012). Direct and Indirect Effects of Media Coverage. Exploring Effects of Presumed Media Influence on Judges, Prosecutors, and Defendants. Studies in Communication/Media, 1(3/4), 473-492.

Kriesi, H.-P. (2001). Die Rolle der Öffentlichkeit im politischen Entscheidungsprozess. Discussion paper P 01-701. Berlin: Wissenschaftszentrum Berlin für Sozialforschung.

Matthes, J. (2013). The Affective Underpinnings of Hostile Media Perceptions: Exploring the Distinct Effects of Affective and Cognitive Involvement. Communication Research 40(3), S. 36087.

Post, S. (2009). Klimakatastrophe oder Katastrophenklima? Die Berichterstattung über den Klimawandel aus Sicht der Klimaforscher. Baden Baden: Nomos.

Post, S. (2015). Incivility in Controversies. The Influence of Presumed Media Influence und Perceived Media Hostility on the Antagonists in the German Conflict over Aircraft Noise. Communication Research (online first, September 2nd 2015)

Reid, S. A. (2012). A Self-Categorization Explanation for the Hostile Media Effect. Journal of Communication, 62(3), 381-399.

Richter, T. (2007). Wie analysiert man Interaktionen von metrischen und kategorialen Prädiktoren? Nicht mit Median-Splits!. Zeitschrift für Medienpsychologie, 19(3), 116-125.

Rojas, H. (2010). „Corrective“ Actions in the Public Sphere: How Perceptions of Media and Media Effects Shape Political Behaviors. International Journal of Public Opinion Research 22(3), $343-$ 63.

Stroud, N. J., Muddiman, A., Lee, J. K. (2014). Seeing Media as Group Members: An Evaluation of Partisan Bias Perceptions. Journal of Communication 64(5), 874-94.

Torgersen, H., Hampel, J. (2012). Calling Controversy: Assessing Synthetic Biology's Conflict Potential. Public Understanding of Science 21(2), 134-48.

Tsfati, Y. (2007). Hostile Media Perceptions, Presumed Media Influence, and Minority Alienation: The Case of Arabs in Israel. Journal of Communication 57(4), 632-51.

Tsfati, Y., \& Cohen, J. (2005). The influence of presumed media influence on democratic legitimacy: The case of Gaza settlers. Communication Research, 32, 794-821.

Vallone, R. P, Ross, L., Lepper, M. R. (1985). The Hostile Media Phenomenon: Biased Perception and Perceptions of Media Bias in Coverage of the Beirut Massacre. Journal of Personality and Social Psychology 49(3), 577-85. 